\title{
Validation of a chloroquine-induced cell death mechanism for clinical use against malaria
}

\author{
J-H Ch'ng ${ }^{\star, 1,2}$, Y-Q Lee ${ }^{1,3,10}$, SY Gun ${ }^{4,10}$, W-N Chia ${ }^{4,10}$, Z-W Chang ${ }^{4,10}$, L-K Wong ${ }^{5}$, KT Batty, ${ }^{6,7}$ B Russell ${ }^{1}$, F Nosten ${ }^{8,9}$, \\ L Renia ${ }^{4}$ and KS-W Tan ${ }^{1}$
}

An alternative antimalarial pathway of an 'outdated' drug, chloroquine (CQ), may facilitate its return to the shrinking list of effective antimalarials. Conventionally, $C Q$ is believed to interfere with hemozoin formation at nanomolar concentrations, but resistant parasites are able to efflux this drug from the digestive vacuole (DV). However, we show that the DV membrane of both resistant and sensitive laboratory and field parasites is compromised after exposure to micromolar concentrations of CQ, leading to an extrusion of DV proteases. Furthermore, only a short period of exposure is required to compromise the viability of late-stage parasites. To study the feasibility of this strategy, mice malaria models were used to demonstrate that high doses of $C Q$ also triggered DV permeabilization in vivo and reduced reinvasion efficiency. We suggest that a time-release oral formulation of $C Q$ may sustain elevated blood CQ levels sufficiently to clear even CQ-resistant parasites.

Cell Death and Disease (2014) 5, e1305; doi:10.1038/cddis.2014.265; published online 26 June 2014

Along with improvements in vector control, surveillance/ diagnosis and treatment accessibility, the development of new drugs to counteract the problem of drug resistance remains integral to the eradication agenda. ${ }^{1}$ Efforts to develop novel antimalarials have been promising, ${ }^{2,3}$ and drugs designed specifically to reverse drug resistance are also being uncovered. ${ }^{4}$ However, novel chemical entities are expensive to test and take considerable time before they can be deployed. In comparison, alternative strategies to fully exploit the existing arsenal of antimalarials (largely already affordable and accessible) are likely to be relatively expedient and cost-effective.

We had previously demonstrated the existence of a novel parasite programmed cell death $(P C D)$ mechanism that was induced by high concentrations of chloroquine (CQ) and shown that clan CA cysteine proteases were key mediators of the pathway. ${ }^{5}$ We had also observed that the permeabilization of the parasite digestive vacuole (DV) was an important upstream trigger of this pathway and that other lysosomotropic compounds that are not parasite-specific could similarly destabilize the DV to initiate parasite PCD. ${ }^{6}$ We hypothesize that by altering the dosing regimen or formulation of $C Q$, it might be possible to reinstate $C Q$ into antimalarial chemotherapy by making use of this novel mechanism. ${ }^{7}$

In this present study, we begin by showing evidence that $\mathrm{CQ}$ treatment is able to result in the extrusion of DV proteases into the parasite cytoplasm. Second, we validate the existence of this PCD pathway in multiple laboratory strains and field isolates to suggest its clinical relevance and universality. Third, we investigate the minimum concentration and duration required for $C Q$ to trigger $P C D$ to determine if the pharmacokinetics of the current $C Q$ regimen might be suitable for initiating PCD. Finally, we make use of two murine malaria models to demonstrate that a short exposure to high levels of $C Q$ is able to induce parasite DV permeabilization in vivo and that this procedure reduces parasite viability.

\section{Results}

Extrusion of plasmepsin IV (PIm-IV) from DV after CQ treatment. To directly link DV permeabilization with the PCD pathway, we began by investigating whether DV proteases were released in response to $C Q$ treatment. We used antibodies to PIm-IV and observed that PIm-IV co-localized clearly with the hemozoin-containing DV in untreated parasites (Figure 1a). In 3D7 trophozoites treated with $3 \mu \mathrm{M}$ of $C Q$ for $4 \mathrm{~h}$, PIm-IV re-localized from the DV to the parasite cytoplasm (Figure 1b). The proportion of cells showing DV localization of PIm-IV decreased markedly from 98 to $21 \%$, whereas those with cytoplasmic distribution of PIm-IV increased from 1 to $73 \%$ (Figures $1 \mathrm{c}$ and d), suggesting that the DV is the likely reservoir of proteases mediating this PCD pathway.

\footnotetext{
${ }^{1}$ Department of Microbiology, National University of Singapore, 5 Science Drive 2, Singapore, Singapore; 'Department of Microbiology, Tumor and Cell Biology, Karolinska Institutet, Nobels väg 16, KI Solna Campus, Box 280, Stockholm, Sweden; ${ }^{3}$ NUS Graduate School for Integrative Sciences and Engineering, National University of Singapore, Centre for Life Sciences (CeLS), \#05-01, 28 Medical Drive, Singapore, Singapore; ${ }^{4}$ Singapore Immunology Network, Agency for Science Technology and Research (A*STAR), Biopolis, 8A Biomedical Grove, Immunos Building, Level 4, Singapore, Singapore; ${ }^{5}$ Department of Chemistry, National University of Singapore, Block S8, Level 3, 3 Science Drive 3, Singapore, Singapore; ${ }^{6}$ School of Pharmacy, Curtin University, GPO Box U1987, Perth Western Australia 6845 Bentley, WA, Australia; ${ }^{7}$ West Coast Institute, 35 Kendrew Crescent, Joondalup, WA, Australia.; ${ }^{8}$ Shoklo Malaria Research Unit, Mahidol-Oxford Tropical Medicine Research Unit, Faculty of Tropical Medicine, Mahidol University, 68/30 Bantung Road, PO.BOX 46, Maesot, TAK, Thailand and ${ }^{9}$ Centre for Tropical Medicine, Nuffield Department of Medicine, University of Oxford, Churchill Hospital, Old Road, Oxford, UK

${ }^{*}$ Corresponding author: J-H Ch'ng, Department of Microbiology, Tumor and Cell Biology (MTC), Karolinska Institutet, Nobels väg 16, KI Solna Campus, Box 280, Stockholm SE-171 77, Sweden. Tel: +46 8 52486202; Fax: +46 8 52487150; E-mail: chngjunhong@ @otmail.com

${ }^{10}$ These authors contributed equally to the work.

Abbreviations: $C Q$, chloroquine; DV, digestive vacuole; PCD, programmed cell death; PIm-IV, plasmepsin IV

Received 17.12.13; revised 04.4.14; accepted 20.5.14; Edited by M Piacentini
} 
a

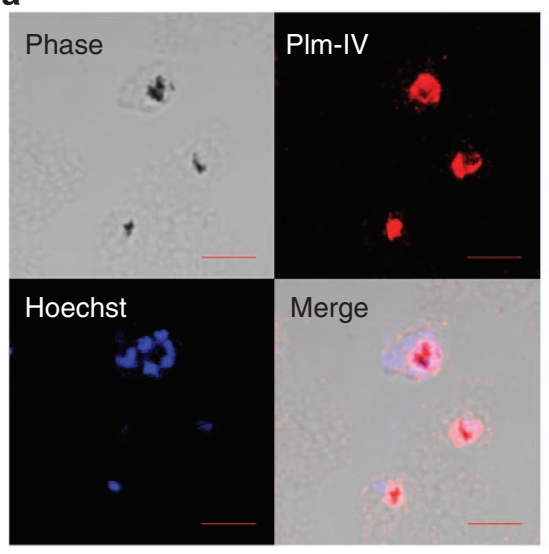

b

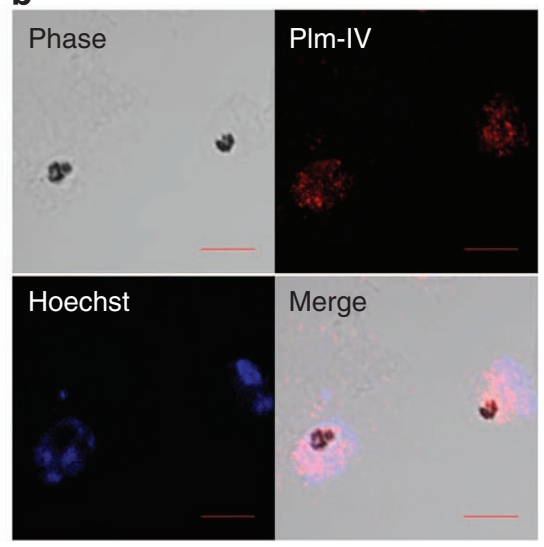

C

\begin{tabular}{|c|c|c|c|c|}
\hline PIm-IV localization & DV & Cytoplasm & Unstained & Total \\
\hline $\begin{array}{c}\text { Vehicle Control } \\
(\%)\end{array}$ & 271 & 2 & 2 & 275 \\
$(98.6 \%)$ & $(0.7 \%)$ & $(0.7 \%)$ & $(100 \%)$ \\
\hline $\mathbf{C Q ~ ( 3 ~} \mu$ M, $\mathbf{4} \mathbf{h r s})$ & 61 & 213 & 16 & 290 \\
$(\%)$ & $(21 \%)$ & $(73 \%)$ & $(6 \%)$ & $(100 \%)$ \\
\hline
\end{tabular}

d

Localization of Plasmepsin IV

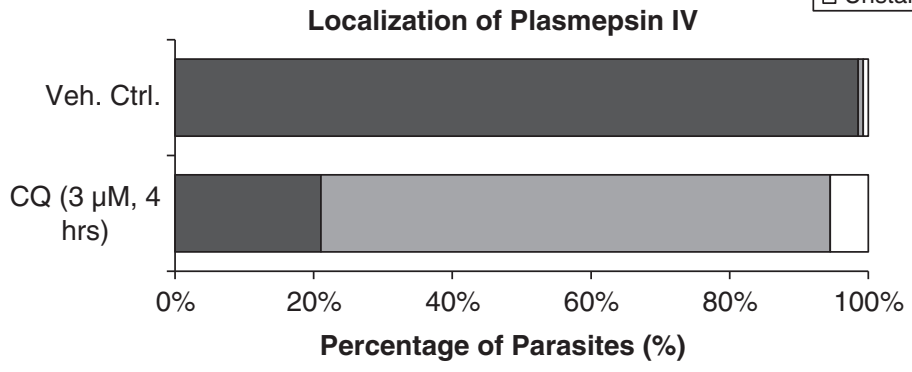

Figure 1 PIm-IV localization assay. (a) Confocal microscopy images show that PIm-IV co-localizes with the dark hemozoin-containing DV in healthy 3D7 P. falciparum parasites but not in (b) parasites treated with $3 \mu \mathrm{M}$ of CQ for $4 \mathrm{~h}$, where PIm-IV staining is less intense and is found throughout the parasite cytoplasm. (Scale bar set at $5 \mu \mathrm{m}$ ). (c) The number of parasites counted and respective percentages, with and without $\mathrm{CQ}$-treatment, and as represented in (d) histogram form, shows a clear redistribution of PIm-IV out of the DV into the cytoplasm

Validation of PCD pathway in multiple laboratory and field parasites. To investigate the clinical relevance of this PCD pathway, six laboratory strains (CQ-sensitive: 3D7 and HB3, CQ-resistant: K1, CS2, Dd2 and T9/94) and six field isolates were assayed (all CQR, artemisinin delayed-clearance: SMRU0233 and SMRU0272). All tested parasites show a significant increase in the proportion of parasites exhibiting these PCD features (all $P<0.05$ ) at higher concentrations of $\mathrm{CQ}$. However, the extent of features varied between strains and the dose at which these changes were observed differed between strains/isolates (Figures $2 a-d$ ). Interestingly, no correlation was observed between the $\mathrm{CQ}$ $I_{50}$ and the concentration at which PCD features became evident. The reduced levels of DNA degradation in 3D7 (half of what was reported previously) ${ }^{5}$ could be due to in suboptimal assaying conditions when working with 12 parasite lines.

CQ-pulse assays for 3D7. To investigate the combined effect of $\mathrm{CQ}$ concentration and duration of exposure, we performed a drug-pulse experiment where parasites were incubated with the stated concentrations of $\mathrm{CQ}$ for various periods before the drug was washed off. Further time was provided for PCD features to manifest or for reinvasion to occur.

Treatment of 3D7 with CQ concentrations of 50 and $100 \mathrm{nM}$ had no measurable effect on $\mathrm{JC}-1$ and Hoechst staining regardless of treatment duration (Figures $2 e$ and f) and did not interfere with reinvasion (Figure $2 \mathrm{~g}$ ). Between $500 \mathrm{nM}$ to $2 \mu \mathrm{M}$ of $C Q$, parasites showed a dose- and duration-dependent loss of JC-1 red staining (Figure $2 \mathrm{e}$ ). Interestingly, DNA degradation, measured by sub-G1 Hoechst staining, appeared to depend more on $\mathrm{CQ}$ concentration than exposure period (Figure 2f). Reinvasion was also clearly inhibited at $500 \mathrm{nM}$ treatment even with only 1-h exposure (Figure $2 \mathrm{~g}$ ) though complete inhibition only occurred beyond $4 \mathrm{~h}$. At $\mathrm{CQ}$ concentrations of $3 \mu \mathrm{M}$ and beyond, the effects of $\mathrm{CQ}$ concentration on JC-1, Hoechst staining and reinvasion was maximal even after only $1-\mathrm{h}$ exposure. Schizont maturation was also not observed. 

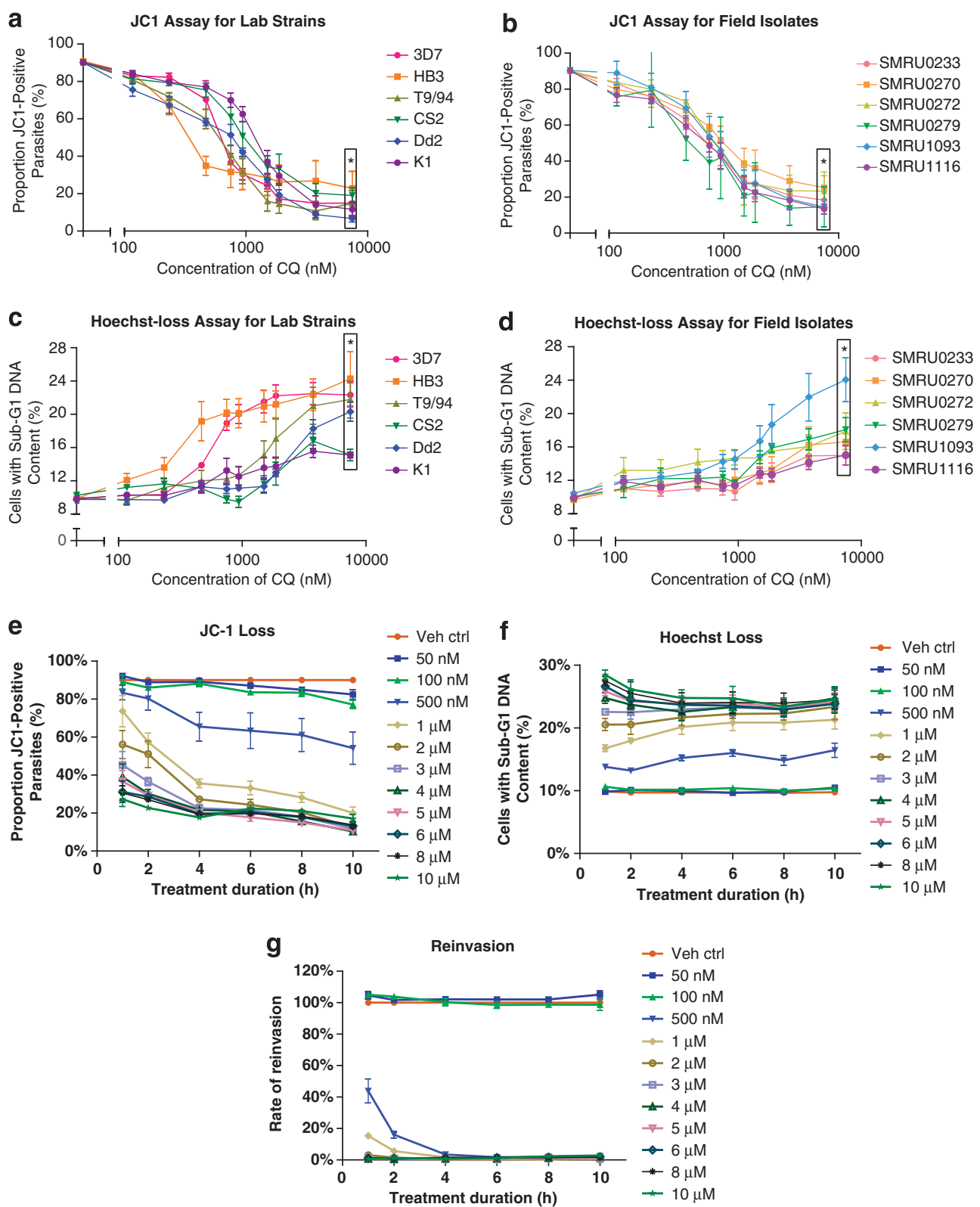

Figure 2 JC-1, Hoechst and reinvasion assays. (a) JC-1 loss occurs in both lab strains and (b) field isolates after $10 \mathrm{~h}$ of high-dose CQ treatment. (c) Similarly, both lab strains and (d) field isolates show signs of DNA degradation as indicated by the reduction of Hoechst staining. ${ }^{*} P$-values $<0.05$ for all points at $7500 \mathrm{nM}$ (boxed) when compared with untreated, with point-specific $P$-values are provided in Supplementary Figure S1. 3D7 parasites were exposed to various doses of CQ for various durations before the drug was washed off and the parasites left to incubate further. After a total of $10 \mathrm{~h},(\mathbf{e}) \mathrm{JC}$-1- and (f) Hoechst-stained parasites were measured while (g) reinvasion efficiency was only measured after a total of $34 \mathrm{~h} .(N=3)$

Ex vivo DV permeabilization of CQ-treated $P$. berghei and $\boldsymbol{P}$. yoelii parasites. To test the possibility of using murine malaria models, $P$. berghei and $P$. yoelii susceptibility to DV permeabilization was assayed using infected blood from hyperparasitemic mice (above $20 \%$ parasitemia). Four hours after freshly drawn blood was spiked with $3 \mu \mathrm{M}$ of $\mathrm{CQ}$ or PBS, cells were stained with Fluo-4AM to determine whether $\mathrm{Ca}^{2+}$ had redistributed out of the DV. Due to difficulties in identifying parasite-infected erythrocytes and visualizing the DV, only late-stage parasites with multiple nuclei and prominent DV were captured.
About 90 and $80 \%$ of healthy $P$. berghei and $P$. yoelii parasites, respectively, showed clear Fluo-4-AM staining of the hemozoin-containing DV (Figures $3 a-c$ ), which for simplicity will be termed as Fluo4AM-positive parasites. In the CQ-treated cells, this proportion were reduced to 60 and $40 \%$, respectively, showing that DV permeabilization also occurs in $P$. berghei and $P$. yoelii parasites after $\mathrm{CQ}$ treatment.

Viability assay of CQ-treated $P$. berghei and $P$. yoelii parasites. Due to difficulty in performing PCD assays 
a i. $P$. berghei (NA)

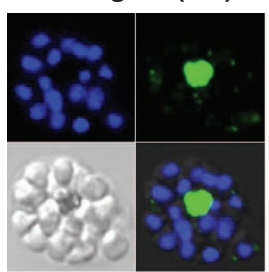

iii. $P$. yoelii (NA)

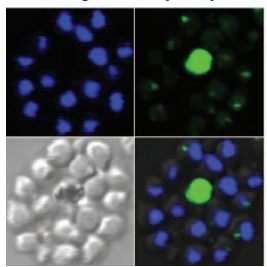

ii. $P$. berghei $(\mathrm{CQ})$

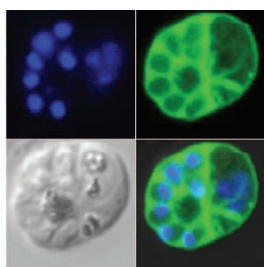

iv. $P$. yoelii (CQ)

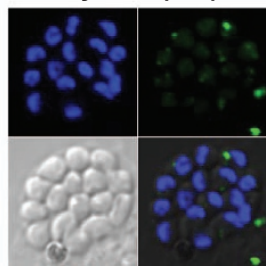

b

\begin{tabular}{|c|c|c|c|c|}
\hline Condition & DV fluorescent & DV not fluorescent & Total Counts & N \\
\hline P. berghei + Veh & $81(93.1 \%)$ & $6(6.9 \%)$ & $87(100 \%)$ & 3 \\
\hline P. berghei $+3 \mu \mathrm{M} \mathrm{CQ}$ & $57(61.3 \%)$ & $36(38.7 \%)$ & $93(100 \%)$ & 3 \\
\hline P. yoelii + Veh & $100(78.7 \%)$ & $27(21.3 \%)$ & $127(100 \%)$ & 3 \\
\hline P. yoelii $+3 \mu \mathrm{M} \mathrm{CQ}$ & $53(41.7 \%)$ & $74(58.3 \%)$ & $127(100 \%)$ & 3 \\
\hline
\end{tabular}

C Effects of $C Q$ on Fluo4AM Staining

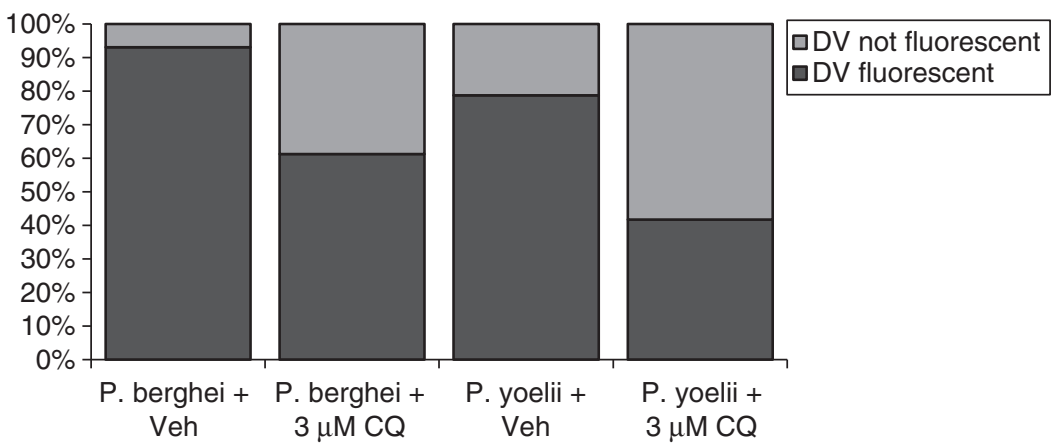

Figure 3 Fluo-4AM staining of $\mathrm{Ca}^{2+}$ in murine malaria parasites. Infected blood was harvested from balb/c mice 14 days postinfection with either $P$. berghei or $P$. yoelii, and magnetically enriched late stages were treated ex vivo with (ai and iii) vehicle control or (aii and iv) $3 \mu \mathrm{M}$ of CQ for $4 \mathrm{~h}$ before Fluo-4AM staining and viewing by confocal microscopy. Cells were (b) enumerated and results are presented as (c) histogram format

(the presence of leukocytes, gametocytes, asynchronous infection, multiple infection and infected reticulocytes having organelles), the viability of CQ-treated schizonts to infect naive mice were assayed instead.

As $P$. berghei schizonts do not rupture/reinvade in vitro, blood from hyperparasitemic mice was matured until they were primarily schizonts. Parasites were exposed to $C Q$ for $4 \mathrm{~h}$ before the drug was washed off and the blood injected into naive mice. In the untreated controls, parasitemia increased almost two-fold by $6 \mathrm{~h}$ postinfection reaching a stable parasitemia by $9 \mathrm{~h}$ (Figure 4a). A similar trend was also observed in parasites treated with $30 \mathrm{nM}$ of $\mathrm{CQ}$ for $4 \mathrm{~h}$, suggesting that this level of drug did not interfere with schizont viability. However, $4 \mathrm{~h}$ after $3 \mu \mathrm{M}$ of $\mathrm{CQ}$, parasitemia declined steadily throughout the first $18 \mathrm{~h}$ and remained low throughout the observed period, indicating that schizont viability was severely compromised.

Schizont maturation could not be performed with $P$. yoelii parasites as their schizonts rupture in vitro. As such, $C Q$ was added directly to freshly isolated (asynchronous) parasites for $4 \mathrm{~h}$ before washing off the drug and infecting naive mice. Parasitemia of mice infected with untreated parasites showed a steady increase in parasitemia (Figure 4b) detectable after $6 \mathrm{~h}$ $(P<0.05)$. A similar increase was detected in mice infected with parasites exposed to $30 \mathrm{nM}$ of $\mathrm{CQ}$ for $4 \mathrm{~h}$. In contrast, parasitemia in mice infected with parasites exposed to $3 \mu \mathrm{M}$ of $\mathrm{CQ}$ only showed a marginal increase in parasitemia after $18 \mathrm{~h}$ $(P<0.05)$. For comparison, an identical experiment with $P$. berghei was performed, and a similar trend was observed with parasites showing a marked reduction in viability after treatment with $3 \mu \mathrm{M} \mathrm{CQ}$ (Figure $4 \mathrm{c}$ ). Although the mixed-stage infection makes it difficult to examine the effects of $C Q$ treatment on any specific parasite stage, the early reduction in parasitemia fold-change (within $9 \mathrm{~h}$ postinfection) suggest that the late-stage $P$. yoelii and $P$. berghei parasites were compromised.

In vivo DV permeabilization of $\boldsymbol{P}$. berghei and $\boldsymbol{P}$. yoelii parasites in CQ-fed mice. To determine whether parasite 
a

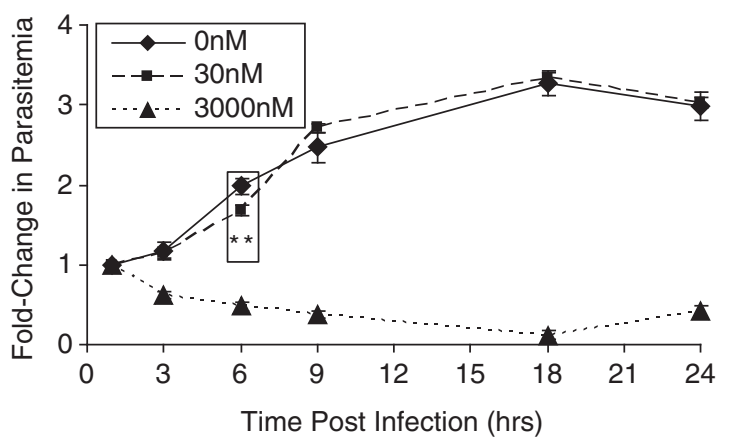

b

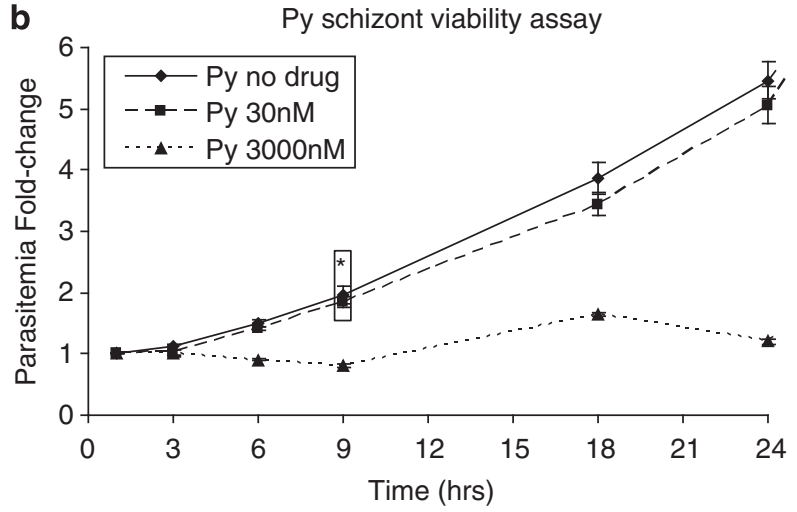

C

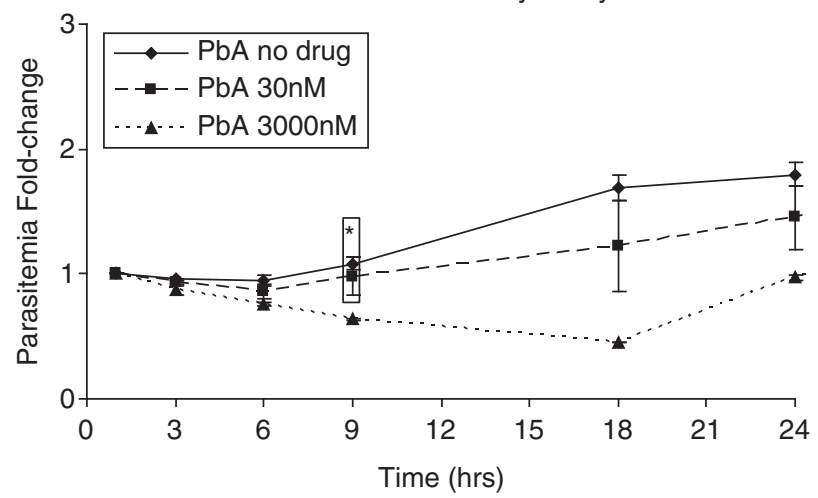

Figure 4 Schizont reinvasion assay. (a) Parasitemia of mice infected with $P$. bergheischizonts matured ex vivo or mixed stages of $(\mathbf{b}) P$. yoeliiand (c) $P$. berghei treated ex vivo with $30 \mathrm{nM}$ or $3000 \mathrm{nM}$ of $\mathrm{CQ}$ for $4 \mathrm{~h}$. ${ }^{*} P$-values $<0.05$ or ${ }^{* *} P$-values $<0.01$ for points (boxed) when compared with respective no-drug control at the same time point. Point-specific $P$-values are provided in Supplementary Figure S2

DV permeabilization occurs in vivo, parasitized blood was obtained from mice $4 \mathrm{~h}$ after they were fed with various doses of $\mathrm{CQ}$ by oral gavage. Once again, parasitized blood from infected mice that were stained with Fluo-4AM ex vivo showed a dose-dependent decrease in the proportion of Fluo4AM-positive $P$. berghei and $P$. yoelii parasites (Figures $5 \mathrm{a}$ and $\mathrm{b}$ ). This decrease was most significant when mice were fed $50 \mathrm{mg} / \mathrm{kg}$ ( $P<0.05$ for both species), suggesting that a higher dose of the drug resulted in a more pronounced effect (Figure $5 \mathrm{c}$ ).

CQ pharmacokinetics in murine model. To correlate the results from the in vivo and ex vivo studies, we assayed for whole-blood levels of $\mathrm{CQ}$ in drug-treated mice. In uninfected mice treated with 10,20 and $50 \mathrm{mg} / \mathrm{kg}$ of $\mathrm{CQ}$, blood $\mathrm{CQ}$ concentrations were approximately 1000,2000 and $5800 \mathrm{nM}$, respectively, after $1 \mathrm{~h}$, and decreased to about 800,700 and $2000 \mathrm{nM}$, respectively, by $4 \mathrm{~h}$ (Figure 6a).

In hyperparasitemic $P$. berghei-infected mice (between 30 and $60 \%$ parasitemia at day 14 postinfection), a more pronounced accumulation of $\mathrm{CQ}$ was detected, reaching about 4700,10640 and $27700 \mathrm{nM}$ after only $1 \mathrm{~h}$ posttreatment with 10,20 and $50 \mathrm{mg} / \mathrm{kg}$ of the drug. After $4 \mathrm{~h}$, blood concentration was 9300,12400 and $23900 \mathrm{nM}$, respectively (Figure 6b). Compared with the uninfected mice, the higher overall blood CQ concentrations was likely due to the drug's accumulation within the parasites. ${ }^{8}$

In hyperparasitemic $P$. yoelii-infected mice (between 20 and $40 \%$ parasitemia at day 14 postinfection), the increased accumulation of $\mathrm{CQ}$ was similarly noted. One hour after being given 10,20 or $50 \mathrm{mg} / \mathrm{kg}$ of the drug, blood CQ concentrations reached 5800, 11400 and $18800 \mathrm{nM}$, respectively (Figure 6c). After $4 \mathrm{~h}$, this level declined to about 5500, 8200 and $14200 \mathrm{nM}$, respectively.

To facilitate comparison with other studies on plasma $\mathrm{CQ}$ levels, plasma levels of $P$. yoelii mice given 10, 20 and $50 \mathrm{mg} / \mathrm{kg}$ of CQ were collected after $4 \mathrm{~h}$ and contained about 200, 330 and $770 \mathrm{nM}$, respectively (results not shown). This corresponded to between 17- and 22-fold reductions from the corresponding whole-blood levels and indicated that $\mathrm{CQ}$ accumulates more within blood cells relative to plasma.

Pharmacodynamic studies of $\mathbf{C Q}$ on parasitemia. To understand the effects of varying $\mathrm{CQ}$ doses on parasite clearance, parasitemia was monitored in $P$. berghei- and $P$. yoelii-infected mice (Figures $6 \mathrm{~d}$ and e). Although all tested doses similarly reduced $P$. berghei parasitemia $9 \mathrm{~h}$ posttreatment (all $P<0.05$ ), only $50 \mathrm{mg} / \mathrm{kg}$ was able to reduce parasitemia within $6 \mathrm{~h}(P<0.05)$, suggesting that a higher dose may elicit a more rapid decrease in parasitemia.

By comparison, inhibition of $P$. yoelii parasitemia appeared unaffected by $\mathrm{CQ}$ treatment until $24 \mathrm{~h}$ where only the 20 and $50 \mathrm{mg} / \mathrm{kg}$ dose showed an appreciable reduction in parasitemia $(P<0.05$ and $P<0.01$, respectively), with the latter having a more pronounced effect.

\section{Discussion}

We had previously characterized a novel PCD pathway in $P$. falciparum that is mediated by clan CA cysteine proteases and suggested that these proteases may originate from the parasite DV. ${ }^{5}$ We also demonstrated that fluorescent-labeled $\mathrm{CQ}$ as well as $\mathrm{Ca}^{2+}$ was able to re-distribute out of the DV in a rapid and abrupt manner and provided ultrastructural evidence of membrane breaching. ${ }^{6}$ In this study, we show that high concentrations of CQ result in the extrusion of PIm-IV out of the DV into the cytoplasm of the parasite, which is in contrast to the classical antimalarial mechanism of $\mathrm{CQ}$ involving the inhibition of hemozoin polymerization and the gradual buildup of toxic by-products. ${ }^{9} \mathrm{PIm}-\mathrm{IV}$ is a $51-\mathrm{kDa}$ aspartic protease that localizes to the parasite DV and is involved in hemoglobin hydrolysis. ${ }^{10,11}$ It appears plausible that other DV proteases, including clan CA cysteine proteases 
a

\begin{tabular}{|c|c|c|c|c|c|}
\hline \multicolumn{7}{|c|}{ Pooled counts from all mice } \\
\hline Species & CQ dose & \# Mice & DV fluorescent & DV not fluorescent & total \\
\hline \multirow{4}{*}{ P. yoelii } & $0 \mathrm{mg} / \mathrm{kg}$ & 4 & 123 & 30 & 153 \\
\cline { 2 - 7 } & $10 \mathrm{mg} / \mathrm{kg}$ & 5 & 107 & 82 & 189 \\
\cline { 2 - 7 } & $20 \mathrm{mg} / \mathrm{kg}$ & 5 & 102 & 101 & 203 \\
\cline { 2 - 7 } & $50 \mathrm{mg} / \mathrm{kg}$ & 5 & 75 & 99 & 174 \\
\hline \multirow{5}{*}{ P. berghei } & $0 \mathrm{mg} / \mathrm{kg}$ & 4 & 105 & 5 & 110 \\
\cline { 2 - 7 } & $10 \mathrm{mg} / \mathrm{kg}$ & 4 & 98 & 22 & 120 \\
\cline { 2 - 7 } & $20 \mathrm{mg} / \mathrm{kg}$ & 4 & 94 & 33 & 127 \\
\cline { 2 - 7 } & $50 \mathrm{mg} / \mathrm{kg}$ & 4 & 72 & 58 & 130 \\
\hline
\end{tabular}

b

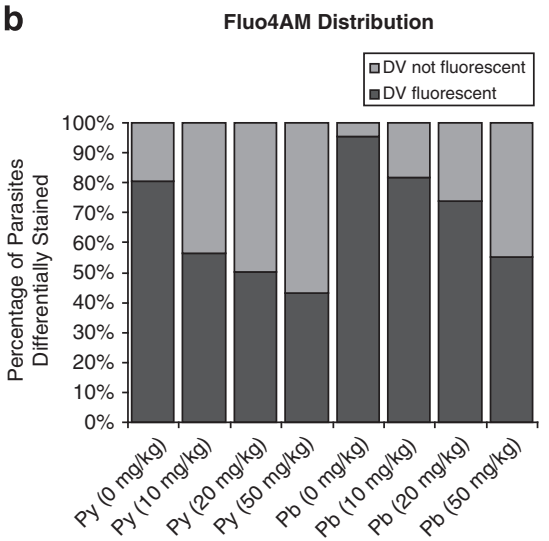

C

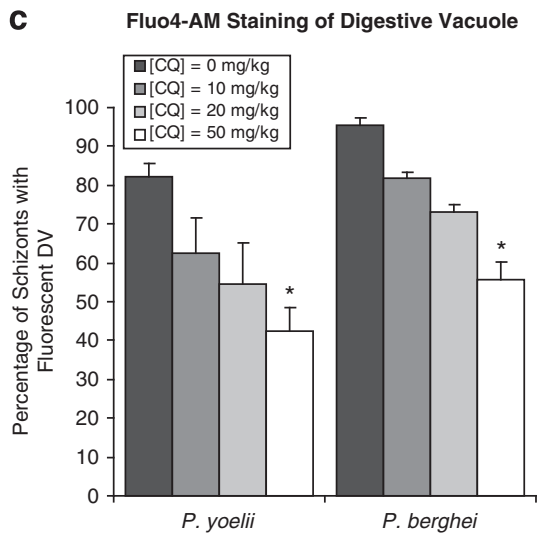

Figure 5 Fluo-4AM staining after $C Q$ administration to infected mice. Mice infected with $P$. berghei or $P$. yoelii were fed with various doses of $C Q$, and blood was harvested after $4 \mathrm{~h}$. After magnetic enrichment for late stages, cells were stained with Fluo-4AM, visualized by confocal microscopy and enumerated. After three experiments, (a) the pooled counts are displayed (b) in histogram. Taking the mean percentage from each experiment, (c) the mean and S.E.M. are shown here. $\left({ }^{*} P<0.05, N \geq 4\right)$

such as the falcipains $(55.8-56.7 \mathrm{kDa})$, could likewise be extruded from the DV to execute PCD.

In addition to the three laboratory strains previously characterized, ${ }^{5}$ the four additional laboratory strains (one CQS and three CQR parasites) and six field isolates from Thailand (all CQR, including two artemisinin-'resistant' delayed-clearance parasites) all exhibited some level of PCD hallmarks, albeit at varying micromolar concentrations, suggesting that this pathway is well conserved in $P$. falciparum and is therapeutically relevant. Though the extent of DNA degradation between strains and isolates tended to vary, this could be due to limitations in simultaneously assaying 12 parasite lines - parasites had varying life-cycle durations and consequently differed in staging at the commencement of experiments. In comparison, the extent of mitochondrial dysregulation was consistent between parasite lines, probably owing to the greater sensitivity of the assay. Heterogeneity in PCD profiles between strains has been previously discussed ${ }^{5}$ and remains the subject of future investigations.

As micromolar concentrations of $\mathrm{CQ}$ are only transient in vivo, ${ }^{12}$ we investigated whether transient high concentrations of $C Q$ were sufficient to trigger parasite $P C D$ and inhibit reinvasion using a drug-pulse assay. Interestingly, micromolar levels of $C Q$ were able to trigger parasite PCD features and inhibit reinvasion even with only 1 -h exposure, whereas nanomolar concentrations did not, even after 10-h exposure. This difference suggests that the 'classical' antimalarial mechanism of $C Q$ that is effected at near $I C_{50}$ levels (inhibition of hemozoin polymerization ${ }^{13}$ ) differs from the mechanism of $\mathrm{CQ}$ at micromolar concentrations, which is likely to be more rapidly injurious. Another study on the stage-dependent sensitivity of $P$. falciparum to $\mathrm{CQ}$ showed similar results, where trophozoites and schizonts exposed to $1 \mu \mathrm{M} \mathrm{CQ}$ for $2 \mathrm{~h}$ had reinvasion inhibited by $60 \%$ and inhibition was complete after 4-h exposure. ${ }^{14}$

To develop an in vivo assay, $P$. berghei and $P$. yoelii parasites were first treated with micromolar CQ ex vivo, and a reduction in the proportion of Fluo-4AM-positive parasites was observed. Interestingly, late stages of both $P$. berghei and $P$. yoelii parasites showed similar changes even though $P$. yoelii is known to exhibit innate resistance to $C Q .{ }^{15}$ Furthermore, there was a dramatic reduction parasite reinvasion, which was very apparent in $P$. berghei (assay performed with schizontsynchronized inoculums) but also detectable in $P$. yoelii (by focusing on the initial reduction of reinvasion). In contrast, nanomolar $\mathrm{CQ}$ exposure showed no effect and suggests that this low level of drug did not have any rapid-onset effect on reinvasion. The association between DV permeabilization and reduced reinvasion in mice malaria parasites is consistent with findings from the $P$. falciparum experiments and suggests that a similar PCD pathway may be involved.

The effects of $C Q$ dosing in vivo was then tested, and mice treated with $50 \mathrm{mg} / \mathrm{kg} \mathrm{CQ}$ showed a significantly larger proportion of $P$. berghei and $P$. yoelii parasites with compromised DV compared with mice that received lower doses. Alterations to the DV membrane in response to escalating doses of $\mathrm{CQ}$ has previously been demonstrated in $P$. berghei in conditions similar to our study, suggesting that high doses of CQ triggers DV dysfunction in vivo. ${ }^{16,17}$

In an attempt to link in vitro, ex vivo and in vivo results, blood $\mathrm{CQ}$ concentrations were assayed in $\mathrm{CQ}$-fed mice. In both $P$. berghei- and $P$. yoelii-infected mice given the $10 \mathrm{mg} / \mathrm{kg}$ dose, 

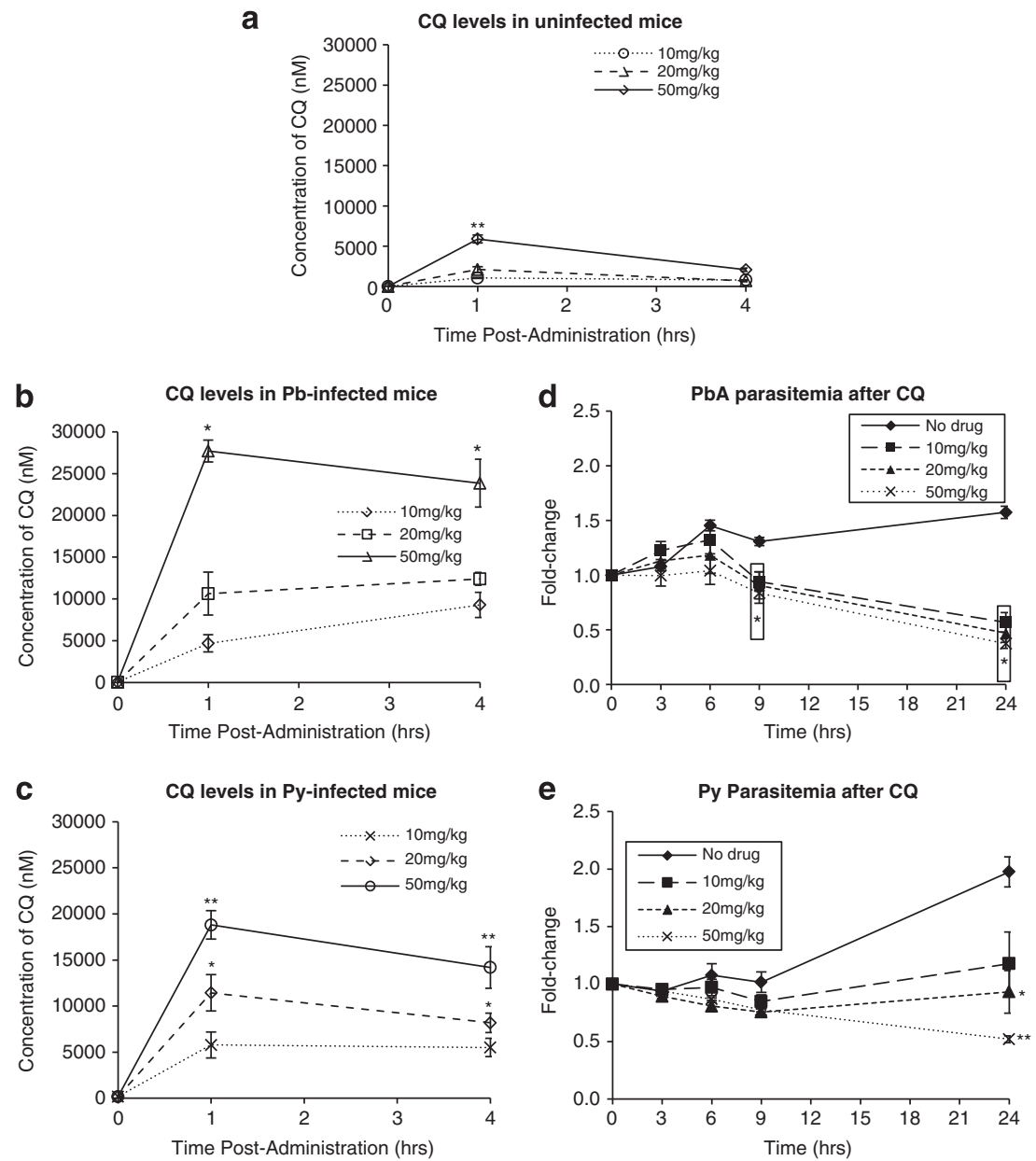

Figure $6 \mathrm{CQ}$ pharmacokinetics and pharmacodynamics. The whole-blood concentration of $C Q$ in $(\mathbf{a})$ uninfected, (b) $P$. berghei-infected and (c) $P$. yoelii-infected mice fed with 10,20 or $50 \mathrm{mg} / \mathrm{kg}$ of $C Q$ at 1 or $4 \mathrm{~h}$ postadministration. ${ }^{*} P<0.05$ or ${ }^{\star \star} P<0.01$ when compared with $10 \mathrm{mg} / \mathrm{kg}$ mice at the same time point, with point-specific $P$-values provided in Supplementary Figure S3. Parasitemia change of mice infected with (d) $P$. berghei or (e) $P$. yoelii after a single oral dose of 10,20 or $50 \mathrm{mg} / \mathrm{kg}$ of CQ. ${ }^{*} P<0.05$ or ${ }^{*} P<0.01$ when compared with no-drug control mice at the same time point, with point-specific $P$-values provided in Supplementary Figure S4

blood levels remained at approximately $5 \mu \mathrm{M}$ for at least $3 \mathrm{~h}$. This value was five-fold more than the corresponding value in uninfected mice, suggesting that a significant amount of $\mathrm{CQ}$ detected may have come from within parasitized erythrocytes. Although whole-blood sampling is generally considered a more accurate measure in clinical pharmacokinetic studies of $\mathrm{CQ},{ }^{12,18,19}$ serum/plasma $\mathrm{CQ}$ concentrations may be more indicative of cell/tissue exposure levels and prove more meaningful in linking in vitro and in vivo findings. Whole-blood CQ levels, at least in mice malaria models, could be affected by differences in hematocrit and parasitemia, both of which can vary significantly depending on parasite species, initial inoculum load and day postinfection. One study showed that blood $\mathrm{CQ}$ concentrations in humans were between 5 and 10-fold higher than in serum, ${ }^{12}$ and another study demonstrated that blood $\mathrm{CQ}$ concentrations in $P$. chabaudi-infected mice with a heavy infection could be 4- to 5-fold higher than mice with light infection. ${ }^{20}$ Taken together, it might be deduced that whole-blood CQ levels could be between 20 and 50 times those of the plasma levels, and indeed, our own assays on a limited number of plasma samples show a blood-to-plasma ratio of about $20: 1$.
Using this estimate, the plasma $\mathrm{CQ}$ levels may be approximated to be around $1 \mu \mathrm{M}$ in both $P$. berghei and $P$. yoelii mice after a single $50 \mathrm{mg} / \mathrm{kg}$ dose, a concentration sufficient to trigger DV permeabilization in vitro. This closely resembles the results from yet another study on $P$. bergheiinfected mice (light infection of 3-4\%) where plasma levels of $\mathrm{CQ}$ after a single $50 \mathrm{mg} / \mathrm{kg}$ intraperitoneal injection was between 500 and $1000 \mu \mathrm{g} / \mathrm{l}$ (approximately, 1.5-3 $\mu \mathrm{M}$ ) during the initial 4-h postadministration. ${ }^{21}$ By the same ratio, plasma CQ levels would be about 200-620 nM after a $10-20 \mathrm{mg} / \mathrm{kg}$ dose, respectively-concentrations insufficient to trigger DV permeabilization in vitro.

To determine whether the decrease in parasites with functional DV corresponded to a decrease in parasite viability, parasitemia was tracked throughout the day in mice treated with 10,20 or $50 \mathrm{mg} / \mathrm{kg}$ of CQ. During the first $6 \mathrm{~h}$ following 10 and $20 \mathrm{mg} / \mathrm{kg}$ of $\mathrm{CQ}$, the increase in $P$. berghei parasitemia suggested that late-stage parasites were still viable and managed to reinvade. Only $9 \mathrm{~h}$ following the drug administration was there a clear reduction in parasitemia. With $50 \mathrm{mg} / \mathrm{kg}$ of $\mathrm{CQ}$, there was negligible increase in parasitemia during the initial $6 \mathrm{~h}$ and parasitemia reduction was evident earlier, 
suggesting that late-stage parasites showed reduced reinvasion propensity at higher blood CQ concentrations. Though the inherent resistance of $P$. yoelii parasites to $\mathrm{CQ}$ has been described, ${ }^{15}$ it is possible that parasites in the present study were inhibited at $50 \mathrm{mg} / \mathrm{kg}$ due to DV permeabilization and associated PCD.

Although dosage escalation is possible in rodent models, the low therapeutic index of $C Q$ means that increasing $C Q$ dosage recommendations need to be cautiously considered. We suggest that a slow-release CQ formulation may permit a gradual increase in blood $C Q$ concentration and sustain these levels sufficiently to trigger parasite PCD while minimizing the risk of toxicity associated with the transient distribution-phase spike in concentration. ${ }^{7} \mathrm{~A}$ study by White et al. ${ }^{22}$ using a continuous CQ infusion of $25 \mathrm{mg} / \mathrm{kg}$ at a rate of $0.83 \mathrm{mg} / \mathrm{kg} / \mathrm{h}$ has shown evidence that whole-blood $\mathrm{CQ}$ levels could be maintained beyond $1000 \mu \mathrm{g} / \mathrm{l}(\sim 3 \mu \mathrm{M})$ for over $10 \mathrm{~h}$ (between 20 and $30 \mathrm{~h}$ posttreatment) without increased side effects. Other studies suggest that smaller repeated oral doses may also achieve a higher blood concentration and increasing drug efficacy without increasing $C_{\text {max }}{ }^{23-25}$ Only in concert with comprehensive pharmacokinetic and pharmacodynamic studies will it be possible to achieve a dosing regimen or drug formulation that will truly maximize the therapeutic effectiveness of $C Q$ and other antimalarials. ${ }^{26}$

Though this approach to exploit the 'unconventional' antimalarial activity of $\mathrm{CQ}$ shows some early promise, it will need to be given in combination with other drugs to prevent the emergence of resistant parasites. In such a context, $\mathrm{CQ}$ may once again find its place in the shrinking arsenal of effective antimalarial drugs if we are able to exploit its alternative antimalarial mechanism of action that can circumvent the current mechanism of drug resistance.

\section{Materials and Methods \\ Parasite culture and $\mathbf{C Q}$ treatment. Six laboratory strains of P. falciparum, 3D7 (MRA-102), HB3 (MRA-155), K1 (MRA-159), CS2 (MRA-96), Dd2 (MRA-156) and T9/94 (MRA-153), were obtained from MR4, ATCC, Manassas, VA, USA. Six $P$. falciparum field isolates were collected from malaria patients attending the clinics of the Shoklo Malaria Research Unit (SMRU) Mae Sod region of Tak Province in the Northwest of Thailand from January 2009 to December 2010 and designated as SMRU0233, SMRU0270, SMRU0272, SMRU0279, SMRU1093 and SMRU1116. $P$. falciparum culture and CQ preparation were described previously. ${ }^{5}$}

Immunofluorescence microscopy for PIm-IV. Thin blood smears of untreated and $\mathrm{CQ}$-induced cultures $(3 \mu \mathrm{M}, 4 \mathrm{~h})$ were fixed in acetone-methanol $(7: 3)$, permeabilized with $0.1 \%$ Triton-X for $10 \mathrm{~min}$ and blocked in $3 \%$ BSA. Mouse monoclonal anti-PIm-IV antibody (1:10 dilution in 3\% BSA) (MRA-814A, MR4, ATCC) was added for $1 \mathrm{~h}$, and slides were washed three times before incubation with Cy3 goat anti-mouse $\operatorname{lgG}(\mathrm{H}+\mathrm{L})$ secondary antibody (1: 1000 dilution, Molecular Probes, Eugene, OR, USA) with Hoechst $33342(0.8 \mu \mathrm{g} / \mathrm{ml})$. At least 250 parasitized erythrocytes were examined by confocal microscopy over three experiments.

Co-staining with JC-1 and Hoechst for laboratory and field parasites. Parasite staining was with JC-1 (Molecular Probes) and Hoechst 33342 (Invitrogen, Eugene, OR, USA) and analysis has been described. ${ }^{6}$

CQ-pulse assay. Trophozoite cultures were incubated with $C Q$ for varying durations before cells were washed twice with MCM and returned to culture. Inclusive of $C Q$ treatment time, cells were incubated for a total of $10 \mathrm{~h}$ to assay for PCD features or $34 \mathrm{~h}$ for reinvasion.
Mice, parasites, experimental infections and drug treatment. Six to twelve weeks old BALB/c mice were bred under specific pathogen-free conditions in the Biomedical Resource Centre, Singapore, Singapore. P. berghei ANKA (clone 15Cy1) and $P$. yoelii yoelii (17X clone 1.1) parasites were maintained by passage through naive mice. Experimental infections were initiated by intraperitoneal injection of $10^{6}$ infected red blood cells from stabilates prepared by passage in C57BL/ $6 \mathrm{~J} \mathrm{mice}$ and stored in liquid nitrogen in Alsever's solution. Parasitemia was monitored by flow cytometry ${ }^{27}$ and/or Giemsa-staining. Blood was drawn by cardiac puncture from mice killed by asphyxiation, collected in a heparinized Vacutainer (BD, Franklin Lakes, NJ, USA) and used immediately after being washed once in $10 \mathrm{ml}$ PBS. Alternatively, smaller quantities of blood $(\sim 100 \mu)$ were obtained via facial bleeding. Administration of $C Q$ was via oral gavage.

Fluo-4AM staining of murine parasites. In ex vivo studies, blood was resuspended to $25 \%$ hematocrit in MCM and then spiked with various concentrations of $\mathrm{CQ}$ for $4 \mathrm{~h}$ at $37^{\circ} \mathrm{C}$. Mature parasites were enriched using magnetic column method ${ }^{28}$ and stained with the Fluo-4-AM as previously described for $P$. falciparum. ${ }^{6}$ For in vivo studies, CQ-fed mice were left for $4 \mathrm{~h}$ before blood was harvested, enriched and stained.

Schizont maturation assay and infection of naive mice. Blood was resuspended in schizont maturation media ${ }^{29}$ supplemented with $20 \%$ FBS for $8 \mathrm{~h}$ at $0.2 \%$ hematocrit. Thereafter, $30 \mathrm{nM}$ or $3 \mu \mathrm{M}$ of $\mathrm{CQ}$ was added for a further $4 \mathrm{~h}$. Cells were washed three times with $50 \mathrm{ml}$ of PBS, and naive mice were then infected by intravenous retro-orbital injection of $0.1 \mathrm{ml}$ of packed cells. For the experiments without schizont maturation, infected blood was resuspended to $2 \%$ hematocrit in MCM, spiked with $C Q$ for $4 \mathrm{~h}$, washed twice with $30 \mathrm{ml}$ PBS and injected. Parasitemia was tracked by FACS as before. ${ }^{27}$

LC-MS sample preparation and separation parameters. Blood from infected and uninfected mice was collected and stored at $-80^{\circ} \mathrm{C}$. For plasma collection, whole blood $(\sim 700 \mu$ l) was centrifuged at $900 \times g$ for $10 \mathrm{~min}$, and $200 \mu \mathrm{l}$ of supernatant was aspirated. Standards were prepared by spiking blood/plasma of infected and uninfected mice with $C Q$ to a final concentration of 30, 150, 300, 1500 and $3000 \mathrm{nM}$. Chromatographic separation was carried out using a RSLC system (Dionex, Germering, Germany) with a Poroshell 300SB-C8 $2.1 \times 75 \mathrm{~mm}^{2}, 5-\mu \mathrm{m}$ column (Agilent, Santa Rosa, CA, USA) at a flow rate of $0.2 \mathrm{ml} / \mathrm{min}$ with $0.5 \%$ acetic acid and acetonitrile as solvents. MS detection utilized micrOTOF-QII mass spectrometer (Bruker, Bremen, Germany). CQ was eluted at approximately $1.2 \mathrm{~min}$. Chromatogram of $\mathrm{CQ}, 320.18 \mathrm{~m} / \mathrm{z}$, was extracted and integrated for all blood/plasma standards (30, 150, 300, 1500 and $3000 \mathrm{nM})$ using the DataAnalysis 4.0 software (Bruker).

Flow cytometry. Cell numbers and fluorescence intensity was assayed using either the BD LSRII Special Order System (BD, San Jose, CA, USA) or CyAn ADP (Beckman Coulter, Fort Collins, CO, USA) as described previously, ${ }^{6}$ and a minimum of 100000 erythrocytes were analyzed.

Statistics. All data shown are mean \pm S.E.M. Statistical difference was analyzed using ANOVA and post-hoc comparison using Tukey's test. For mice experiments, the Kruskall-Wallis test was used. Significantly different results $(P \leq 0.05)$ were highlighted.

Ethics. Blood collection for malaria culture was approved by Institutional Review Board (IRB) of the National University of Singapore (NUS-IRB Reference Code: 11-383, Approval Number: NUS-1475). Clinical field isolates were collected following ethical guidelines in approved protocols: OXTREC Reference Number 29-09 (Center for Clinical Vaccinology and Tropical Medicine, University of Oxford, Oxford, UK). Use of field isolates was in accordance with NUS IRB (Reference Code: 12-369E). All animal experiments and procedures were approved by the Institutional Animal Care and Use Committee (IACUC) of $A^{*}$ STAR (Biopolis, Singapore) (Authorization No. IACUC no.110630) in accordance with the guidelines of the Agri-Food and Veterinary Authority (AVA) and the National Advisory Committee for Laboratory Animal Research (NACLAR) of Singapore.

\section{Conflict of Interest}

The authors declare no conflict of interest. 
Acknowledgements. We thank Benoit Malleret and Carla Claser for their input to experimental design. We thank all of the patients and staff of the SMRU for their contribution to this study. SMRU is sponsored by the Wellcome Trust of Great Britain, as part of the Oxford Tropical Medicine Research Program of Wellcome Trust-Mahidol University. We also thank the MR4 as part of the BEl Resources Repository, NIAID, NIH from whom the following reagents were obtained: Plasmodium falciparum 3D7, MRA-102, deposited by DJ Carucci; $P$. falciparum HB3, MRA-155, deposited by TE Wellems; $P$. falciparum K1, MRA-159, deposited by DE Kyle; $P$. falciparum Dd2, MRA-156, deposited by TE Wellems; $P$. falciparum CS2, MRA-96, deposited by SJ Rogerson; P. falciparum T9/94, MRA-153, deposited by D Walliker; and P. falciparum 13.9.2, MRA-814A, deposited by DE Goldberg (anti-Plasmepsin IV monoclonal antibody). This study was supported by generous grants awarded to KS-WT from the National Medical Research Council, Singapore (NMRC/EDG/1038/2011 and NMRC/1310/2011). Part of the study was supported by core grant to SIgN and from the Horizontal Programme on Infectious Diseases under the Agency for Science, Technology and Research (A*STAR, Singapore).

\section{Author contributions}

Study design: J-HC and KS-WT; PCD assays: J-HC (laboratory, field and murine parasite studies), Y-QL (CQ-pulse studies); LC-MS analyses: J-HC and L-KW; animal handling: WNC, SYG and Z-WC; field isolates: BMR and FN; pharmacokinetics and pharmacodynamics: J-HC and KTB; manuscript preparation: all authors.

1. The malERA Consultative Group on Drugs. A research agenda for malaria eradication: drugs. PLOS Med 2011; 8: e1000402.

2. Yeung BK, Zou B, Rottmann M, Lakshminarayana SB, Ang SH, Leong SY et al. Spirotetrahydro beta-carbolines (spiroindolones): a new class of potent and orally efficacious compounds for the treatment of malaria. J Med Chem 2010; 53: 5155-5164.

3. Burrows JN, Burlot E, Campo B, Cherbuin S, Jeanneret S, Leroy D et al. Antimalarial drug discovery - the path towards eradication. Parasitology 2013; 17: 1-12.

4. Ch'ng JH, Mok S, Bozdech Z, Lear MJ, Boudhar A, Russell B et al. A whole cell pathway screen reveals seven novel chemosensitizers to combat chloroquine resistant malaria. Sci Rep 2013; 3: 1734

5. Ch'ng JH, Kotturi SR, Chong AG, Lear MJ, Tan KS. A programmed cell death pathway in the malaria parasite Plasmodium falciparum has general features of mammalian apoptosis but is mediated by clan CA cysteine proteases. Cell Death Dis 2010; 1: e26.

6. Ch'ng JH, Liew K, Goh AS, Sidhartha E, Tan KS. Drug-induced permeabilization of parasite's digestive vacuole is a key trigger of programmed cell death in Plasmodium falciparum. Cell Death Dis 2011; 2: e216.

7. Ch'ng JH, Renia L, Nosten F, Tan KS. Can we teach an old drug new tricks? Trends Parasitol 2012; 28: 220-224.

8. Macomber PB, O'Brien RL, Hahn FE. Chloroquine: physiological basis of drug resistance in Plasmodium berghei. Science 1966; 152: 1374-1375.

9. Zhang Y, Hempelmann E. Lysis of malarial parasites and erythrocytes by ferriprotoporphyrin IX-chloroquine and the inhibition of this effect by proteins. Biochem Pharmacol 1987; 36: 1267-1273.

10. Banerjee R, Liu J, Beatty W, Pelosof L, Klemba M, Goldberg DE. Four plasmepsins are active in the Plasmodium falciparum food vacuole, including a protease with an active-site histidine. Proc Natl Acad Sci USA 2002; 99: 990-995.

11. Rosenthal PJ. Falcipains and other cysteine proteases of malaria parasites. Adv Exp Med Biol 2011; 712: 30-48.

12. Ducharme J, Farinotti R. Clinical pharmacokinetics and metabolism of chloroquine. Focus on recent advancements. Clin Pharmacokinet 1996; 31: 257-274.

13. Foley M, Tilley L. Quinoline antimalarials: mechanisms of action and resistance and prospects for new agents. Pharmacol Ther 1998; 79: 55-87.
14. Yayon A, Vande Waa JA, Yayon M, Geary TG, Jensen JB. Stage-dependent effects of chloroquine on Plasmodium falciparum in vitro. J Protozool 1983; 30: 642-647.

15. Warhurst DC, Killick-Kendrick R. Spontaneous resistance to chloroquine in a strain of rodent malaria (Plasmodium berghei yoelii). Nature 1967; 213: 1048-1049.

16. Warhurst DC, Hockley DJ. Mode of action of chloroquine on Plasmodium berghei and P. cynomolgi. Nature 1967; 214: 935-936.

17. Macomber PB, Sprinz H. Morphological effects of chloroquine on Plasmodium berghei in mice. Nature 1967; 214: 937-939.

18. Rombo L, Ericsson O, Alvan G, Lindstrom B, Gustafsson LL, Sjoqvist F. Chloroquine and desethylchloroquine in plasma, serum, and whole blood: problems in assay and handling of samples. Ther Drug Monit 1985; 7: 211-215.

19. Krishna S, White NJ. Pharmacokinetics of quinine, chloroquine and amodiaquine. Clinical implications. Clin Pharmacokinet 1996; 30: 263-299.

20. Cambie G, Verdier F, Gaudebout C, Clavier F, Ginsburg H. The pharmacokinetics of chloroquine in healthy and Plasmodium chabaudi-infected mice: implications for chronotherapy. Parasite 1994; 1: 219-226.

21. Moore BR, Page-Sharp M, Stoney JR, llett KF, Jago JD, KT. Batty. Pharmacokinetics, pharmacodynamics, and allometric scaling of chloroquine in a murine malaria model. Antimicrob Agents Chemother 2011; 55: 3899-3907.

22. White NJ, Miller KD, Churchill FC, Berry C, Brown J, Williams SB et al. Chloroquine treatment of severe malaria in children. Pharmacokinetics, toxicity, and new dosage recommendations. N Engl J Med 1988; 319: 1493-1500.

23. Ursing J, Kofoed PE, Rodrigues A, Bergqvist Y, Rombo L. Chloroquine is grossly overdosed and overused but well tolerated in Guinea-bissau. Antimicrob Agents Chemother 2009; 53: 180-185.

24. Ursing J, Kofoed PE, Rodrigues A, Blessborn D, Thoft-Nielsen R, Bjorkman A et al. Similar efficacy and tolerability of double-dose chloroquine and artemether-lumefantrine for treatment of Plasmodium falciparum infection in Guinea-Bissau: a randomized trial. J Infect Dis 2011; 203: 109-116.

25. Pussard E, Lepers JP, Clavier F, Raharimalala L, Le Bras J, Frisk-Holmberg M et al. Efficacy of a loading dose of oral chloroquine in a 36-hour treatment schedule for uncomplicated Plasmodium falciparum malaria. Antimicrob Agents Chemother 1991; 35: $406-409$

26. Bakshi RP, Nenortas E, Tripathi AK, Sullivan DJ, Shapiro TA. Model system to define pharmacokinetic requirements for antimalarial drug efficacy. Sci Transl Med 2013; 5: 205ra135.

27. Malleret B, Claser C, Ong AS, Suwanarusk R, Sriprawat K, Howland SW et al. A rapid and robust tri-color flow cytometry assay for monitoring malaria parasite development. Sci Rep 2011; 1 : 118.

28. Spadafora C, Gerena L, Kopydlowski KM. Comparison of the in vitro invasive capabilities of Plasmodium falciparum schizonts isolated by Percoll gradient or using magnetic based separation. Malar J 2011; 10: 96

29. Ecker A, Lakshmanan V, Sinnis P, Coppens I, Fidock DA. Evidence that mutant PfCRT facilitates the transmission to mosquitoes of chloroquine-treated Plasmodium gametocytes. J Infect Dis 2011; 203: 228-236.

(1) (2) Cell Death and Disease is an open-access journal published by Nature Publishing Group. This work is licensed under a Creative Commons Attribution-NonCommercialShareAlike 3.0 Unported License. The images or other third party material in this article are included in the article's Creative Commons license, unless indicated otherwise in the credit line; if the material is not included under the Creative Commons license, users will need to obtain permission from the license holder to reproduce the material. To view a copy of this license, visit http://creativecommons.org/ licenses/by-nc-sa/3.0/ 